\title{
A catalog of rotational and radial velocities for evolved stars
}

\section{Southern stars ${ }^{\star}, \star \star$}

\author{
J. R. De Medeiros ${ }^{1}$, S. Alves ${ }^{1}$, S. Udry ${ }^{2}$, J. Andersen ${ }^{3,4}$, B. Nordström ${ }^{3}$, and M. Mayor ${ }^{2}$ \\ ${ }^{1}$ Departamento de Física, Universidade Federal do Rio Grande do Norte, Campus Universitário, 59072-970 Natal, RN, Brasil \\ e-mail: renan@dfte.ufrn.br \\ 2 Observatoire de Genève, Université de Genève, Chemin des Maillettes 51, 1290 Sauverny, Switzerland \\ 3 The Niels Bohr Institute, University of Copenhagen, Juliane Maries Vej 30, 2100 Copenhagen, Denmark \\ ${ }^{4}$ Nordic Optical Telescope, Apartado 474, 38700 Santa Cruz de La Palma, Spain
}

Received 19 November 2012 / Accepted 10 December 2013

\begin{abstract}
Rotational and radial velocities have been measured for 1589 evolved stars of spectral types F, G, and K and luminosity classes IV, III, II, and Ib, based on observations carried out with the CORAVEL spectrometers. The precision in radial velocity is better than $0.30 \mathrm{~km} \mathrm{~s}^{-1}$ per observation, whereas rotational velocity uncertainties are typically $1.0 \mathrm{~km} \mathrm{~s}^{-1}$ for subgiants and giants and $2.0 \mathrm{~km} \mathrm{~s}$ for class II giants and Ib supergiants.
\end{abstract}

Key words. stars: late-type - stars: fundamental parameters - binaries: spectroscopic - techniques: radial velocities - catalogs stars: evolution

\section{Introduction}

Over the past two decades, observations have been carried out at the Geneva Observatory, Switzerland, and the Federal University of Rio Grande do Norte, Brazil, to accurately measure projected rotational velocities $(v \sin i)$ of evolved stars, with the aim of studying the evolution of stellar rotation with stellar age.

The technique used is to combine a high-resolution spectrometer with the cross-correlation technique, which yields accurate, high $\mathrm{S} / \mathrm{N}$ cross-correlation line profiles from relatively low $\mathrm{S} / \mathrm{N}$ spectra. From these profiles, accurate radial velocities and, once calibrated, projected rotational velocities $(v \sin i)$ with an accuracy better than $1 \mathrm{~km} \mathrm{~s}^{-1}$ can be derived, allowing measurements of $v \sin i$ for large samples of relatively faint stars with telescopes of moderate aperture.

Most of the observations presented here were made with the CORAVEL cross-correlation spectrometers (Baranne et al. 1979). In addition, De Medeiros et al. (2006) measured rotational velocities, $v \sin i$, for 100 metal-poor stars with the digital version of the cross-correlation procedure, using spectra obtained with the FEROS (Kaufer \& Pasquini 1998) and CORALIE (Baranne et al. 1996) spectrometers.

As part of this programme, De Medeiros \& Mayor (1999) measured $v \sin i$ for 1541 stars of luminosity classes IV, III, and II, De Medeiros et al. (2002b) presented $v$ sin $i$ for $232 \mathrm{Ib}$ supergiant stars, and De Medeiros et al. (2004) also measured $v \sin i$ for 78 double-lined binaries with an evolved component. These high-quality data have inspired several studies,

\footnotetext{
* Based on observations collected at the Haute-Provence Observatory, Saint-Michel, France, and at the European Southern Observatory, La Silla, Chile.

$\star \star$ Table 1 is only available at the CDS

via anonymous ftp to cdsarc.u-strasbg. fr $(130.79 .128 .5)$ or via http://cdsarc.u-strasbg.fr/viz-bin/qcat?J/A+A/561/A126
}

enabling reliable investigations of stellar rotational characteristics in different regions of the $\mathrm{H}-\mathrm{R}$ diagram (Carlberg et al. 2011; Cortés et al. 2009; Melo et al. 2001), the relationship between rotation and different stellar properties (Monaco et al. 2011; Raghavan et al. 2010; López-Santiago et al. 2010; Grunhut et al. 2010; Massarotti et al. 2008; De Medeiros et al. 2002a; Cutispoto et al. 2002), constraints on theoretical models (Eggenberger et al. 2010; Brun \& Palacios 2009) and in many studies on extra-solar planets (e.g. Watson et al. 2010; Döllinger et al. 2009).

The present work brings complementary results for our observational efforts, with the measurements of projected rotational velocity $v \sin i$ for southern subgiant, giant, bright giant, and Ib supergiant stars of spectral types F, G, and K, listed in the Bright Star Catalog (Hoffleit \& Jaschek 1982; Hoffleit et al. 1983). Although the primary aim of this investigation is to study the rotational behaviour of evolved stars, our observational procedure also produced a large set of radial velocity measurements, representing an important tool for answering several questions in stellar astrophysics, including the search for planets around evolved stars.

This paper is arranged as follows. Section 2 presents the definition of the sample, the observational procedure used throughout this survey, and the calibration of rotational velocities, with a discussion of their probable errors. The list of individual $v \sin i$ measurements and mean radial velocities are presented in Sect. 3.

\section{The observational programme}

The present sample consists of a total of 1702 mainly southern F, $\mathrm{G}$, and $\mathrm{K}$ stars of luminosity classes IV, III, II, and Ib listed in the Bright Star Catalog. Most of these stars were observed in different programmes carried out at the Geneva Observatory, 
Table 2. Double-lined spectroscopic binary systems SB2 with evolved component.

\begin{tabular}{|c|c|c|}
\hline HD & $(B-V)$ & ST \\
\hline 352 & 1.38 & $\mathrm{~K} 2 \mathrm{III}+\mathrm{F}$ \\
\hline 5357 & 0.38 & F4III \\
\hline 5516 & 0.94 & G8IIIb \\
\hline 6397 & 0.41 & F4II-III \\
\hline 7788 & 0.47 & F6IV \\
\hline 8949 & 1.12 & K1III \\
\hline 10308 & 0.44 & F2III \\
\hline 12641 & 0.88 & G5II-III+G5V \\
\hline 13480 & 0.78 & $\mathrm{G} 5 \mathrm{III}+\mathrm{F} 5 \mathrm{~V}$ \\
\hline 16920 & 0.40 & F4IV \\
\hline 17904 & 0.41 & F4IV \\
\hline 18894 & 0.60 & G0IV-V \\
\hline 24546 & 0.41 & F5IV \\
\hline 29104 & 0.754 & G5II-III+A-F \\
\hline 34029 & 0.80 & G5IIIe+G0III \\
\hline 35798 & 1.11 & K1III \\
\hline 38751 & 1.01 & G8III \\
\hline 39040 & 1.12 & K1III \\
\hline 40084 & 1.23 & G5III \\
\hline 41116 & 0.82 & G7III \\
\hline 43358 & 0.46 & F5IV: \\
\hline 46178 & 1.07 & KOIII \\
\hline 47415 & 0.53 & F8IV \\
\hline 47703 & 0.49 & F8III \\
\hline 52973 & 0.79 & F7-G3Ib \\
\hline 58972 & 1.43 & K3-IIIFe-0.5 \\
\hline 59148 & 1.11 & K2III \\
\hline 59878 & 1.01 & K0II-III+F8V \\
\hline 60318 & 1.01 & K0III \\
\hline 63799 & 1.12 & K1III \\
\hline 64235 & 0.41 & F5IV \\
\hline 68461 & 0.89 & G8III \\
\hline 71622 & 0.90 & G8III \\
\hline 73596 & 0.401 & F5III \\
\hline 75629 & 0.95 & G8III \\
\hline 76143 & 0.42 & F5IV \\
\hline 78418 & 0.66 & G5IV-V \\
\hline 80586 & 0.93 & G8III-IV+F5V \\
\hline 81873 & 1.04 & K0III \\
\hline 82543 & 0.62 & F7IV-V \\
\hline 83808 & 0.49 & F6II+A1-5V \\
\hline 92787 & 0.33 & F5III \\
\hline 98993 & 1.46 & K6III \\
\hline 106677 & 1.14 & K0IIIe+K0IIIe \\
\hline 109312 & 0.46 & F3III-IV \\
\hline 120064 & 0.49 & G5III+A7V: \\
\hline 120672 & 0.48 & F6IV-V \\
\hline 122703 & 0.45 & F5III \\
\hline 123999 & 0.54 & F9IV w \\
\hline 125383 & 0.92 & G8III \\
\hline 129502 & 0.38 & F2III \\
\hline 133340 & 1.01 & G8III \\
\hline 139862 & 0.94 & G7.5IIIaFe-0.5 \\
\hline 150682 & 0.40 & F2III \\
\hline 158614 & 0.72 & G9IV-VHdel 1 \\
\hline 159870 & 0.59 & G5III+A5V \\
\hline 163506 & 0.34 & F2Ib \\
\hline 164136 & 0.39 & F2II \\
\hline 169268 & 0.34 & F6III-IV \\
\hline 171802 & 0.37 & F5III \\
\hline 172088 & 0.55 & F9IV \\
\hline 172103 & 0.42 & F1IV-V \\
\hline 174881 & 1.18 & K1II-III \\
\hline 178619 & 0.52 & F5IV-V \\
\hline 179094 & 1.09 & K1IV \\
\hline
\end{tabular}

Table 2. continued.

\begin{tabular}{rcc}
\hline \hline HD & $(B-V)$ & ST \\
\hline 179950 & 0.56 & G8:III+A8V \\
185734 & 0.97 & G8III-IV \\
196753 & 0.98 & K0II-III+A3V \\
198084 & 0.54 & F8IV-V \\
200497 & 0.68 & G4III \\
201051 & 1.05 & K0II-III \\
201772 & 0.44 & F5IV+F6V \\
202447 & 0.53 & G0III+A5V \\
204960 & 1.04 & K1III \\
205539 & 0.35 & F0IV \\
205877 & 0.60 & F7III \\
206901 & 0.43 & F5IV \\
210334 & 0.72 & G2IV+K0III \\
218527 & 0.91 & G8III-IV \\
\hline
\end{tabular}

Table 3. Evolved stars with no CORAVEL dip.

\begin{tabular}{cc}
\hline \hline HD & ST \\
\hline 432 & F2III-IV \\
571 & F2II \\
4338 & F1IVn \\
6763 & F0III-IV \\
6953 & K7III \\
11522 & F0IIIn \\
13305 & F4III \\
20121 & F7III+A0V \\
23010 & F5II \\
34658 & F5II \\
37788 & F0IV \\
56986 & F2IV \\
57852 & F0-2IV-V \\
65925 & F5III \\
72779 & G0III \\
77601 & F6II-III \\
91942 & K3-4II \\
96202 & F3IV \\
104827 & F0IV-V \\
109085 & F2III-IV \\
126251 & F4III \\
127739 & F2IV \\
144183 & F2II \\
164259 & F2IV \\
171237 & F3II-III \\
186357 & F1III \\
187038 & K3III \\
194943 & F2IV \\
200723 & F3IV \\
203784 & F6II-III \\
203842 & F5III \\
207958 & F1III \\
208177 & F5IV \\
219571 & F1III \\
\hline &
\end{tabular}

the majority devoted to studying stellar binarity (Duquennoy et al. 1991; De Medeiros \& Mayor 1999; De Medeiros et al. 2002 b 2004) or to precise measurements of radial velocity in programmes on Galactic structure (Andersen et al. 1985; Prevot et al. 1985; Maurice et al. 1987; Nordström et al. 2004a).

As in previous papers (De Medeiros \& Mayor 1999; De Medeiros et al. 2002b, 2004), the observations reported here were made using the two CORAVEL spectrometers (Baranne et al. 1979) mounted on the 1.54-m Danish telescope at ESO, 
La Silla (Chile), and the 1-m Swiss telescope at Haute-Provence Observatory, Saint Michel (France). Radial velocities were derived by direct cross-correlation of the stellar spectra with a binary $(0,1)$ physical template, constructed from the spectrum of the K2 III star Arcturus and mounted inside the spectrometers. The radial-velocity system applied is that defined by Udry et al. (1999). Typical integration times were $5 \mathrm{~min}$, and data was reducted using standard procedures (Duquennoy 1987; Duquennoy et al. 1991; De Medeiros \& Mayor 1999). For a complete discussion of the observational procedure, calibration, and error analysis, readers are referred to Duquennoy (1987), Duquennoy et al. (1991), and De Medeiros \& Mayor (1999).

Here, we just recall a few salient points. In all cases, the radial velocity uncertainty is derived from an instrumental error added in quadrature to photon and scintillation noise, which are estimated using the computed parameters of the crosscorrelation profiles (Baranne et al. 1979). Different studies of large data samples (Duquennoy et al. 1991; Udry et al. 1997; De Medeiros \& Mayor 1999) show that the typical uncertainty for CORAVEL radial velocity is about $0.3 \mathrm{~km} \mathrm{~s}^{-1}$ for slowly rotating stars, generally with $v \sin i<20 \mathrm{~km} \mathrm{~s}^{-1}$. For faster rotators, the uncertainty is somewhat greater.

Rotational velocities $(v \sin i)$ were obtained through an appropriate calibration of the widths of cross-correlation profiles, as described by De Medeiros \& Mayor (1999). The original $v \sin i$ calibration by Benz \& Mayor (1984) is also valid for subgiant and giant stars of luminosity classes IV and III, but for class II and Ib bright giants and supergiants, the increase in macroturbulence with spectral type required a new calibration of the width of the cross-correlation profile into $v \sin i$ as measured from a Fourier transform of line profiles from Gray \& Toner (1986, 1987). Whereas Benz \& Mayor (1984) obtained for the parameter associated with the CORAVEL crosscorrelation profiles $\sigma_{0}$ the value of $6.88 \mathrm{~km} \mathrm{~s}^{-1}$ for stars of luminosity classes V to III, the new calibration shows that for the luminosity classes II and Ib the value of $\sigma_{0}$ is $7.158 \mathrm{~km} \mathrm{~s}^{-1}$ and $7.978 \mathrm{~km} \mathrm{~s}^{-1}$, respectively.

The computed $v \sin i$ has a typical uncertainty of around $1.0 \mathrm{~km} \mathrm{~s}^{-1}$ for subgiant and giant stars with $v \sin i \ll 30 \mathrm{~km} \mathrm{~s}^{-1}$, whereas for bright giants and $\mathrm{Ib}$ supergiants, we conservatively assume an uncertainty of $2.0 \mathrm{~km} \mathrm{~s}^{-1}$, since it is impossible to define precise limits between rotation and macroturbulence. For faster rotators, those with $v \sin i$ higher than $30 \mathrm{~km} \mathrm{~s}^{-1}$, De Medeiros \& Mayor (1999) estimate an uncertainty of about $10 \%$, regardless of luminosity class.

\section{Contents}

The main results of this catalogue are listed in Table 1, which presents CORAVEL rotational and mean radial velocities for 1589 evolved FGK stars of luminosity classes IV, III, II, and $\mathrm{Ib}$, ordered by HD number, for single stars and single-lined spectroscopic binaries. Columns are as follows:

1. HD number;

2. spectral type;

3. $(B-V)$ color index;

4-5. mean radial velocity RV and its uncertainty $\epsilon$, on $N$ number of CORAVEL observations. In this case, the uncertainty is given by $\max \left(\epsilon_{1} / \sqrt{N}, \sigma / \sqrt{N}\right)$, where $\epsilon_{1}$ is the typical error for one single radial velocity measurement;

6. radial velocity dispersion (rms) $\sigma$;

7. $E / T$, the ratio of observed to expected rms dispersion for observations, when $N \geq 2$;
8. $P\left(\chi^{2}\right)$, the probability that the radial velocity of the star is constant;

9. $N$, number of observations for each star;

10. time span $\Delta T$ of observations;

$11-12$. rotational velocity $V \sin i$ and its uncertainty $\epsilon_{\text {rot }}$;

13. remarks. The remarks SBO and SB indicate, respectively, single-lined spectroscopic binaries for which orbital parameters are available in the literature, and stars displaying single-lined spectroscopic binary behaviour. Nevertheless, for a few stars classified as SB, the RV variability may reflect another cause, e.g. pulsation.

A number of the programme stars, identified in Table 1, were already included in the papers by Andersen et al. (1985), Prevot et al. (1985), and Maurice et al. (1987), with radial velocities referred to in the 1985 standard system of Udry et al. (1999), while the velocities listed here are referred to in the revised zero-point of Udry et al. (1999). The colour-dependent differences from the earlier velocities are small, about $0.11,0.31$, and $0.45 \mathrm{~km} \mathrm{~s}^{-1}$ for F-, G-, and K-type stars, respectively, but can be noticed in precise work.

Table 2 lists 79 SB2 and SB3 binary systems also observed by CORAVEL, many of which were detected here for the first time, while Table 3 presents the evolved F-type stars for which no correlation dip was obtained with CORAVEL. These are undoubtedly fast rotators.

The individual radial velocity measurements for single and SBO stars, as well as for SB not included in follow-up programmes, are available at the Centre de Données Astronomiques de Strasbourg (CDS).

Acknowledgements. We gratefully acknowledge the support of the CORAVEL observers in this survey, particularly Gilbert Burki, Bernard Pernier, Pierre North, Jean-Claude Mermilliod and Gerard Jasniewicz. We also thank Emile Ischi and Bernard Tartarat for technical maintenance of the CORAVELs and B. L. Canto Martins, I. C. Leão and J. D. do Nascimento for assistance in preparing tables and data controls. S. Alves acknowledges a graduate fellowship and a PNPD fellowship from the CAPES Brazilian agency. This study used the SIMBAD database, operated at the CDS in Strasbourg, France, and it was supported by continuous grants from the Swiss National Science Foundation. Research activities of the Observational Stellar Board of the Federal University of Rio Grande do Norte are supported by the Brazilian agencies CNPq, FAPERN, and the INCT INEspaço. The observations at the Danish $1.54 \mathrm{~m}$ telescope from ESO, La Silla, Chile, was supported by grants from ESO and Danish observing time, and financially by the Danish Natural Science Research Council through the Danish Board for Astronomical Research.

\section{References}

Andersen, J., Nordstrom, B., Ardeberg, A., et al. 1985, A\&AS, 59, 15 Baranne, A., Mayor, M., \& Poncet, J. L. 1979, Vist. Astron., 23, 279 Baranne, A., Queloz, D., Mayor, M., et al. 1996, A\&AS, 119, 373

Benz, W., \& Mayor, M. 1984, A\&A, 138, 183

Brun, A. S., \& Palacios, A. 2009, ApJ, 702, 1078

Carlberg, J. K., Majewski, S. R., Patterson, R. J., et al. 2011, ApJ, 732, 39

Cortés, C., Silva, J. R. P., Recio-Blanco, A., et al. 2009, ApJ, 704, 750

Cutispoto, G., Pastori, L., Pasquini, L., et al. 2002, A\&A, 384, 491

De Medeiros, J. R., \& Mayor, M. 1999, A\&AS, 139, 433

De Medeiros, J. R., Da Silva, J. R. P., \& Maia, M. R. G. 2002a, ApJ, 578, 943

De Medeiros, J. R., Udry, S., Burki, G., \& Mayor, M. 2002b, A\&A, 395, 97

De Medeiros, J. R., Udry, S., \& Mayor, M. 2004, A\&A, 427, 313

De Medeiros, J. R., Silva, J. R. P., Do Nascimento, J. D., Jr., et al. 2006, A\&A, 458,895

Döllinger, M. P., Hatzes, A. P., Pasquini, L., Guenther, E. W., \& Hartmann, M. 2009, A\&A, 505, 1311

Duquennoy, A. 1987, A\&A, 178, 114

Duquennoy, A., Mayor, M., \& Halbwachs, J.-L. 1991, A\&AS, 88, 281

Eggenberger, P., Miglio, A., Montalban, J., et al. 2010, A\&A, 509, A72

Gray, D. F., \& Toner, C. G. 1986, ApJ, 310, 277

Gray, D. F., \& Toner, C. G. 1987, ApJ, 322, 360 
Grunhut, J. H., Wade, G. A., Hanes, D. A., \& Alecian, E. 2010, MNRAS, 408, 2290

Hoffleit, D., \& Jaschek, C. 1982, The Bright Star Catalogue (New Haven, USA: Yale University Observatory)

Hoffleit, D., Saladyga, M., \& Wlasuk, P. 1983, A Supplement to the Bright Star Catalogue (New Haven, USA: Yale University Observatory)

Kaufer, A., \& Pasquini, L. 1998, Proc. SPIE, 3355, 844

López-Santiago, J., Montes, D., Gálvez-Ortiz, M. C., et al. 2010, A\&A, 514, A97

Massarotti, A., Latham, D. W., Stefanik, R. P., \& Fogel, J. 2008, AJ, 135, 209

Maurice, E., Andersen, J., Ardeberg, A., et al. 1987, A\&AS, 67, 423

Mayor, M., \& Maurice, E. 1985, in Stellar Radial Velocities, eds. A. G. D. Philip, \& D. W. Latham (Schenectady: L. Davis Press), Proc. IAU Colloq., 88, 299
Melo, C. H. F., Pasquini, L., \& De Medeiros, J. R. 2001, A\&A, 375, 851

Monaco, L., Villanova, S., Moni Bidin, C., et al. 2011, A\&A, 529, A90

Nordström, B., Mayor, M., Andersen, J., et al. 2004a, A\&A, 418, 989

Nordström, B., Mayor, M., Andersen, J., et al. 2004b, VizieR Online Data Catalog, V/117

Prevot, L., Andersen, J., Ardeberg, A., et al. 1985, A\&AS, 62, 23

Raghavan, D., McAlister, H. A., Henry, T. J., et al. 2010, ApJS, 190, 1

Udry, S., Mayor, M., Andersen, J., et al. 1997, Proc. of the ESA Symp., Hipparcos - Venice '97, ESA SP, 402, 693

Udry, S., Mayor, M., \& Queloz, D. 1999, in Precise Stellar Radial Velocities, IAU Colloq., 170, ASP Conf. Ser., 185, 367

Watson, C. A., Littlefair, S. P., Collier Cameron, A., Dhillon, V. S., \& Simpson, E. K. 2010, MNRAS, 408, 1606 